\title{
Business Value of Integrated BIM-based Asset Management
}

\author{
Mustapha Munir, Arto Kiviniemi, Stephen W. Jones
}

\begin{abstract}
Purpose - Currently, Building Information Modelling (BIM) is largely seen as a 3D-model, not as an information model or information management tool. This wrong perception of BIM and low interest in 3D Asset Management (AM) is one of the major reasons for the slow adoption by clients in the Architectural, Engineering, and Construction (AEC) industry. The paper aims to identify the techniques and strategies of streamlining AM systems for BIM-based integration, and how the information is captured from physical assets towards BIM-based integration for clients to derive value from BIM investments.
\end{abstract}

Design/methodology/approach - A qualitative case study strategy was used to study the strategic implementation process of integrating BIM with AM systems and the business value of BIM in AM by a large asset owner in the United Kingdom (UK).

Findings - The paper identifies key strategies in the adoption of BIM-based processes by an asset owner, the implementation process, the challenges and the benefits attained. Several barriers were identified as challenges of adopting BIM-based processes in AM: 1) Complexity and cost associated with BIM; 2) Irrelevance of 3D-geometric data in AM processes; 3) Nature of asset ownership structure; 4) Managing the asset handover process; 5) Managing change within the organisation. Organisations will have to consider the following issues in streaming asset information with BIM: 1) The development for a clear strategy prior to adoption; 2) Connecting the strategy to the business goals; 3 ) Conducting the discovery exercise to identify organisational information needs.

Originality - The research addresses a significant gap in the development of techniques and strategies for asset owners to streamline BIM with AM systems and derive business value from such integration. The research context is a case study involving a large owner-operator in the UK that has been able to derive value from BIM systems in their AM processes. The key value of the paper is improving asset owners' understanding of BIM in AM by demonstrating the implementation strategies, linkage to organisational objectives, challenges, value management process and business value of BIM in AM. Another contribution of the paper is improving the understanding of BIM, which is usually viewed as 3D-models and that 3D geometric data does not have much value for AM tasks.

Paper type - Case study

KEYWORDS: BIM, AM, Assets, Integration, Asset owners, Value 


\subsection{INTRODUCTION}

Increasingly, the Architectural, Engineering, Construction (AEC) industry is tasked with the manufacture, collection, and utilisation data for critical decision making on assets. The demand for more interpretable asset data by public and private sector asset owners has been on the increase, with many taking on the initiative to implement BIM in the lifecycle of their investments (Love et al., 2013). Aside from data, the utilisation of BIM in Asset Management (AM) has also transitioned from the use of BIM as a software to an asset information management system (Love et al., 2013). All these changes are happening as a result of the paradigm shift in the perception of AM by asset owners from necessary maintenance of assets to asset optimisation.

BIM is a methodology that can assist asset managers to manage their portfolios efficiently and effectively. However, the integration of BIM with AM systems, the management process, strategies and protocols continue to present a major challenge to BIM implementation across building lifecycle phases (Jupp, 2013). The slow adoption of BIM in AM may be as a result of the nature of BIM, which may be more suited to the design and construction stages. Also, another factor is the seeming complexity of utilisation of BIM for the operations and use phase. The overcoming of these barriers and adoption of BIM will provide the opportunity for asset managers to integrate all the disparate silos of asset information contained in different systems into a federated information model that will facilitate easy access and analysis.

For most asset owners, AM tasks are essential to their business processes. As such, asset maintenance forms part of the strategic business objectives, and asset maintenance data is linked to other strategic systems within the business for asset planning and AM tasks. These organisations rely on structured and trusted data for critical decision-making (Brous et al., 2015). Generally, it is a reality that most organisations generate far more data than they possibly use; yet, at the same time, they do not have all the data they actually need (Lin et al., 2008). The challenge for asset owners is having streamlined data infrastructure and polices, which will deliver the needed high-quality and up-to-date data for business processes and critical decision making. 
Asset managers have attempted for many years to create AM systems which produce quality data but end up having data that are embedded with significant amount of meaningless data or missing required information (Lin et al., 2008; Brous et al., 2015). Public sector organisations are facing increasing challenges in the management of their assets and many owner-operators are looking for ways to optimise their assets in order to improve the efficiency of their AM processes through data-driven decision-making (Brous et al., 2015).

The context of this research is to investigate and identify the strategies and techniques of how asset owners can integrate BIM with $\mathrm{AM}$ systems and the business value to be derived from such integration. This study analyses the process of a large owner-operator organisation in the United Kingdom (UK) that utilises BIM in AM tasks. Also, it demonstrates how asset owners can identify what information needs to be captured from physical assets towards BIM-based integration. It can be said that most asset owners do not understand how to go about the whole process of integrating these systems in order to derive real business value from BIM-based processes in AM. 


\subsection{LITERATURE REVIEW}

\subsection{BIM-BASED ASSET MANAGEMENT}

Firstly, there is need to define an asset and AM within the context of this study. An asset is any tangible or intangible item of value owned by an organisation. Furthermore, Schneider et al. (2006) define AM as 'operating a group of assets over the whole technical life-cycle, guaranteeing a suitable return while ensuring defined service and security standards'. Similarly, PAS 55-2 (BSI, 2008) views AM as 'systematic and coordinated activities and practices through which an organisation optimally and sustainably manages its assets and asset systems, their associated performance, risks and expenditures over their life cycles for the purpose of achieving its organisational strategic plan'. The practice of AM involves the creation, acquisition, maintenance, operation, renewal, rehabilitation and disposal of assets. Generally, AM is about understanding risks and developing good business strategies through appropriate organisational processes and technological models to manage an asset (Mohseni, 2003). On the other hand, BIM is defined as a tool, technique and methodology of 'generating, storing, managing, exchanging, and sharing building information in an interoperable and reusable way' (Vanlande et al., 2008).

Similarly, BIM-based AM is the use of object-based information models and management techniques as decision support tools in digital asset information management to monitor and evaluate asset data for the purpose of asset optimisation. The main characteristics of BIM-based AM processes are those that relate to data management, storage, sharing and exchange in AM tasks, not 3D geometry as often thought in the context of BIM. These processes facilitate the utilisation of an asset information database to enable asset owners to enhance their operations and optimise the management of their assets.

A number of studies have focused on the implementation of BIM in the operations and use phase of built assets. Cavka et al. (2017) developed a technique to formalise owner information requirements. The definition and capture of these operational requirements will aid the asset owner in the transition from BIM to Facilities Management (FM) processes right through the handover of a facility. Whilst the study helps to inform asset owners in organising their requirements, it does not focus on the organisational-level strategic objectives of BIM implementation in AM. Similarly, Pishdad-Bozorgi et al. 
(2018) reported on the transition to BIM-based FM during handover of a facility in a realworld case study. The study identifies: 1) the need for a clear definition of BIM-FM scope; 2) data collection through projects phases; and 3) smooth interoperability between BIM and FM tools; as criteria for successful implementation of BIM by an asset owner. Even though the study presents operational workflows for the management of information throughout a project's lifecycle, it however does not suggest any strategic approach for an asset owner at the AM level. Ashworth et al. (2016) developed an FM to BIM concept model that outlines the process of preparing organisational BIM strategies based on Employer Information Requirements (EIR). The study proposes how the facility manager can be integrated in the lifecycle project development of a built asset and how these processes link to the project phases but it does not show how the EIR is captured. On the other hand, Love et al. (2014) identified four significant aspects for asset owners to proactively manage the BIM implementation process in order to realise value from their investments. They are; governance, change management, stakeholder management and performance measurement. Although the framework is conceptual in nature, this study adopts this perspective as a starting point with the understanding that if the four aspects of the framework are effectively managed during the planning and implementation of BIM in AM, an asset owner will be able to derive business value from BIM-based processes.

\subsection{BIM-AM SYSTEMS AND DATA GOVERNANCE}

Essentially, data generated from AM systems is only valuable to the organisation if users are able to interpret it into useful information within a particular context or for a specific task (Brous et al., 2016). Love et al. (2014) suggest the need for asset owners to develop a governance perspective where BIM is integrated with projects, programmes and portfolios throughout the asset's lifecycle. Most organisations are unable to exploit the large amounts of available asset data into meaningful management information to enhance their operations (Lin et al., 2008). This failure has led to the manifestation of silos of information in owner-operator organisations. Hence, asset managers have found it very difficult to utilise real-time asset data in their business operations. Also, asset managers need to be aware of complexities brought about by interoperability and ensure compatibility between systems, such as asset register systems, work order management systems and condition monitoring systems, when adopting BIM-based solutions for AM. 
Lin et al. (2007) suggest some classifications within the context of governance and data management for asset owners based on technological, organisational, and people perspectives. Similarly, Brous et al. (2015) researched the governance of data in a major owner-operator organisation. The study points out the need for the establishment of a department that is responsible for asset information management within the organisation. Furthermore, Brous et al. (2015) highlighted the utilisation of Constructive Objects and the INtegration of processes and Systems (COINS) standard for BIM-based integration within the organisation. COINS is characterised by an object tree which ensures that GIS, 2D drawings, 3D models, IFC models and object type library are linked and stored in a database (igure 1).

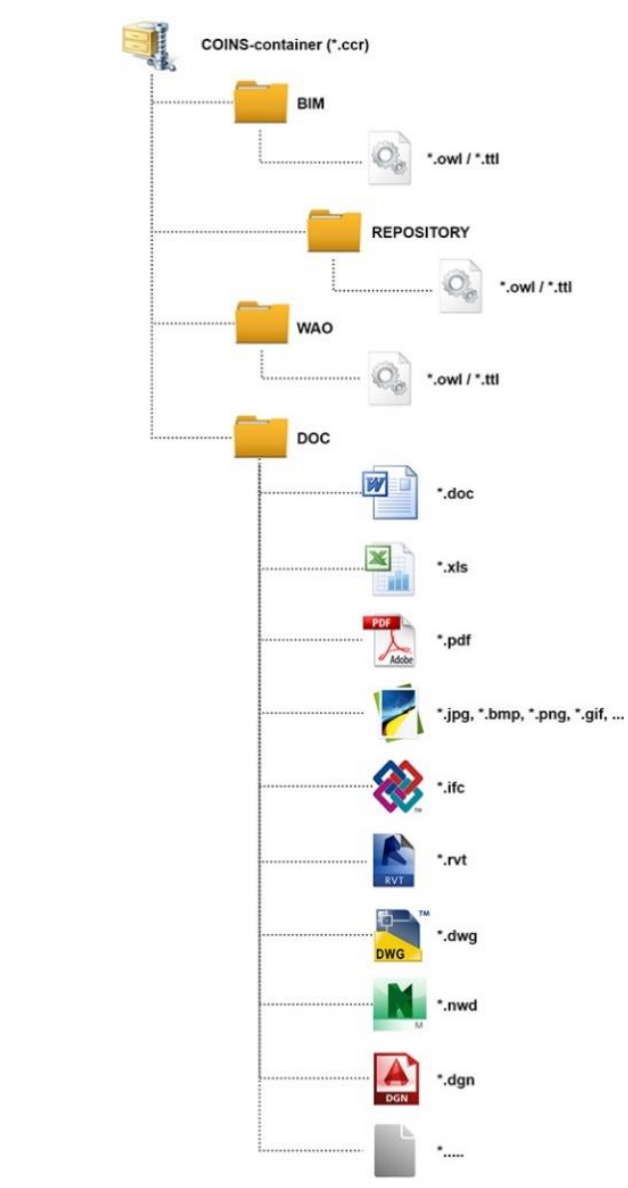

Figure 1: COINS container folder structure (DBIC, 2015)

Brous et al. (2015) goes on to propose a framework for asset owners to break down the silos of information and unlock trusted information to flow freely to where it is required in the organisation. The framework assesses how data governance affects completeness, 
consistency, accuracy, relevancy, and timeliness of data. The study helps demonstrate effective systems and data governance for asset owners. In the same manner, Campos et al. (2017) developed a framework on how asset owners can make well-organised decisions, plan and analyse data for AM functions through effective data governance. The framework is in three layers: 1) IT technologies and data sources; 2) Data analysis; 3) Data visualisation; which, is aimed at assisting asset owners to convert operational data into information and knowledge that will result in increased business value.

\subsubsection{ASSET REGISTER}

An asset register contains all the assets owned by an organisation. Details of the value, type, function and location of assets are specified in the asset register. For most organisations, the asset register is the core element of their AM systems because it holds the database of information regarding every asset. The register facilitates the quick retrieval of asset information by managers for daily operations.

Despite the significance of the asset register and its role in AM, there is no industry standard that could guide asset managers through the whole process of developing the standard classification of assets within the register. ISO 27001 (2013) only provides a framework for asset owners to develop the inventory of assets, nomination of asset owners, definition of asset use, and safe and secure management of information.

The asset register may have two parts; financial and maintenance asset register. The financial asset register contains information that relates to the current worth of assets, and the total ownership costs. This could be the purchase cost of assets, current value of assets after depreciation, operating and maintenance costs. On the other hand, the maintenance section of the asset register provides a structure for handling a wide range of information that is required to support the maintenance of assets. This is the asset type, asset capacity, drawings, specifications, manuals, suppliers, maintenance instructions, maintenance history and failure history. Therefore, asset managers must ensure that records are reconciled between the financial asset register and maintenance asset register. 


\subsubsection{ASSET HIERARCHY}

Asset hierarchy is the technique for classifying assets into groups in an asset register. The asset hierarchy can be based on asset type or asset function or both. The purpose of the hierarchy is to provide a framework for collecting and reporting asset information through some procedural standards in an organisation. The hierarchy denotes different levels at which asset information can be reviewed for critical decisions. Similarly, asset hierarchy management is a process of tracking every asset and their associated location in an organisation. Usually, this represents family tree type relationship of the asset portfolio. The assets are drawn to denote relationships between assets and constituent elements such as spaces, equipment, processes and physical locations. These techniques of management classifications make it easier for asset managers to collect data, analyse and plan for asset interventions.

Most asset owners operate with informal asset hierarchy systems (Lin et al., 2008). This leads to administrative problems with incorrect or insufficient data for critical decision making. Some of the key asset aspects of asset hierarchy classification are asset class, asset group, asset type and asset parameters. Asset hierarchy systems can have several levels of categorisations based on asset number, equipment number, location, type, function and process. The naming is usually clearly defined with consistent naming formats. The use of consistent standard naming conventions has enabled asset managers to integrate their registers with computerised systems, thus, enabling the organisation to conduct speedy searches of asset information within the AM system. For example, AB 01 BF AHU36 may represent; AB- Ancillary Building, 01 - Space Reference, BF - Basement Floor, AHUN - Air Handling Unit, Asset Number - 36.

\subsection{BIM CHANGE MANAGEMENT}

The adoption of BIM-based processes for AM tasks by any owner-operator will involve a shift in organisational culture. This change in culture will bring about risks and challenges that must be managed to ensure successful implementation of new systems and subsequent attainment of organisational objectives. Good change management practices have to be adopted for asset owners to benefit from the opportunities and avoid threats that will be faced by their organisation when implementing BIM-based processes. Change 
management in this context refers to the technique and methodology put in place by an asset owner in order to achieve the desired outcome of a BIM initiative or project.

Due to the technological and organisational changes involved, proactive change management is required for asset owners transitioning from traditional AM systems to one supported by BIM (Jupp and Awad, 2017). The transition is usually constrained by the lack of consistent approaches. Jupp and Awad (2017) discuss two change management strategies for BIM implementation in the operations and use phase, which are; the deployment of a 'niche project with follow up', and an 'overall step-by-step' approach. On the other hand, Lewin (1951) propose a three-step model to organisational change management. They are; unfreeze, moving and refreezing. Similarly, Kotter (1996) propose an eight-step technique for managing organisational change. They are; to establish a sense of urgency about the need to achieve change; create a guiding coalition; develop a vision and strategy; communicate the change vision; empower broad-based action; generate short-term wins; consolidate gains and produce more change; and anchor new approaches in the corporate culture.

\subsection{BIM STAKEHOLDER MANAGEMENT}

BIM provides opportunity for multi-disciplinary collaboration where all parties can effectively participate during project delivery (Eastman et al., 2011). This approach provides the opportunity for asset managers, facility managers, end-users and contractors to participate because of improved shared understanding during asset development and subsequent asset operation and maintenance. These stakeholders will have the potential to retrieve, update and modify asset information right through BIMbased processes. With this opportunity comes other risks or challenges to manage successfully the contributions of all parties within a BIM-based environment. Stakeholder management in this context refers to the tools and techniques used to successfully deliver a BIM project through active engagement of project participants.

To assist this process, there exists collaboration platforms that help manage the activities of stakeholders during BIM implementation. There are a number of vendors that provide software-based collaboration platforms for AM tasks such as Common Data Environments (CDE), Computerised Maintenance Management Systems (CMMS) and 
Integrated Workspace Management Systems (IWMS), Computerised Asset Management Software (CAMS), Computer Aided Facilities Management (CAFM) and BIM (Codinhoto and Kiviniemi, 2014; Guillen et al., 2016; Love et al., 2016). These products allow stakeholders to monitor asset performance, activity and parameters during routine tasks. Thus, improving communication between stakeholders during project execution.

\subsection{BIM PERFORMANCE MANAGEMENT}

BIM-based processes will not only enhance the management of assets, but rather, they will provide an efficient mechanism for good information management throughout the organisation. Performance management in this context involves the set of managerial activities put in place to observe, monitor and evaluate organisational performance with the sole aim of attaining pre-set BIM-based objectives of good information management and benefits realisation. The success of the entire BIM process depends on the weakest contributor to the system (Walasek and Barszcz, 2017). The goals and targets of BIM implementation should be clearly defined early in the process by the asset owner. In the same manner that a BIM manager is utilised during the construction process, the role of an information manager will have to be established for the BIM-based AM processes. The information manager will be responsible for the efficient and effective management of asset data and ensure interoperability in line with organisational and project objectives.

For business process performance to be measured, the BIM-based processes will have to be evaluated. This evaluation will enable asset owners to identify the productivity improvements that result from the implementation of BIM-based processes (Succar et al., 2012). Managing expectations is important during this process. Asset owners need to be realistic and not set ambitious targets that are not achievable. Also, another issue is that BIM adopters usually expect to realise significant benefits while they are still inexperienced users (Succar et al., 2012). Better understanding of the BIM-based processes, the systems workings, and potential impact on the organisation will help managers during the initial stages of implementation.

To evaluate organisational BIM performance, Succar et al. (2012) identified five components for asset owners to consider, they are; BIM capability stages, BIM maturity levels, BIM competencies, organisational scales, and granularity levels. BIM capability 
refers to the basic ability of an asset owner operator to perform BIM-based processes to execute AM tasks. The BIM capability stages represent the major milestones an owneroperator needs to attain in order to implement BIM-based processes continuously whilst improving on the previous stage. BIM maturity relates to the quality, repeatability and degree of excellence of BIM-based processes within a BIM capability towards executing AM tasks. This is the level of versatility an organisation possesses within a certain capability stage. BIM competency in an organisation refers to the individual competencies for assessing capability and maturity that can be classified into technology, process and policy (Succar et al., 2012). Organisational scales refer to the classifications of business sector, company size and markets. Lastly, granularity levels are assessment criteria grouped to the enhance BIM capability. 


\subsection{METHODOLOGY AND RESEARCH QUESTION}

\subsection{RESEARCH QUESTIONS}

The study addresses the following research questions:

- What are the techniques and strategies of streamlining AM systems for BIM-based integration?

- What information should be captured from physical assets towards BIM-based integration and how?

\subsection{RESEARCH METHODS}

The case study involved a large retail organisation in the UK to investigate how asset owners can formulate and implement a strategy to streamline their AM systems within BIM. The study also investigates the business value derived through BIM-based processes and the challenges experienced by the asset owner. The owner-operator organisation will be referred to as 'Company $\mathrm{X}$ ' in this study.

The study focuses on the AM activities carried out by the Estates Department under the Property Division in Company X. This department is responsible for maintaining over 2,000 built assets at the strategic level, with a gross floor area of over $3,000,000 \mathrm{~m}^{2}$. These comprise of trading and non-trading assets owned or managed by Company X. The department is also responsible for identifying strategic approaches, technologies and innovations for implementation within the organisation. The department also liaises with the FM department within the organisation to manage the physical assets at the operational level.

The research adopts a case study strategy. This strategy is suitable for studying a contemporary phenomenon within a real-life context (Yin, 2003). The case study strategy provides an in-depth investigation into particular instances within the research subject (Fellows and Liu, 2015). The study is exploratory and descriptive in nature. Exploratory research methods are suitable for seeking new insights to assess phenomena in a new light and descriptive research methods are appropriate in portraying the accurate characteristics of events or situations (Saunders et al., 2012). The study adopts an operational construct sampling technique as a typology of purposive sampling for the 
identification of the case and selection of participants for data collection. This sampling strategy is good for studying real world examples of events or phenomena (Patton, 2002). Company X was selected purposefully because the population of BIM adopters in AM in the UK was not known, and as a result, random sampling was not an option. Furthermore, the study partly utilised the snowball sampling (Patton, 2002) to explore the authors' network in order to identify and select the case study including the participants. This strategy was used because of the rare cases of operational BIM implementation in AM. The criteria used for selecting participants were:

- Participants have advanced level of knowledge and understanding of BIM in AM.

- Participants were senior staff of the owner operator.

- Participants were senior staff of the consulting company engaged for the project.

- Participants are key stakeholders in the project.

- Participants are high level decision makers in the project.

- Participants are involved in every process of strategic and operational decision making.

Participant validation was also conducted on the collected data (Saunders et al., 2012). The analysed results were sent back to the participants for authentication of the accuracy of data presented in this study. Also, data triangulation validity was utilised through the use of multiple sources of data in the study (Patton, 2002).

The study is divided into two phases. The first phase is the literature review, where the study explores existing research on BIM and data governance in AM. The second phase is the case study research, where the study investigates the techniques and strategies of integrating BIM-AM systems in Company X. The review of literature was conducted to identify relevant studies related to data governance and asset management practices. Also, key aspects related to this subject matter such as governance, change management, performance management and stakeholder management were reviewed. The study identified frameworks and methodologies developed by other studies within the area of BIM implementation in AM. The second phase has two main activities; they are: interviews and archival analysis. Interviewing as a data collection technique was utilised for this study (Patton, 2002). This technique was used to gather in-depth information on the processes involved by Company X in integrating BIM with AM systems. The purpose of the interviews was to extract qualitative descriptions of organisational strategies, 
business process re-engineering techniques, change management strategies, business value and challenges of implementing BIM-based processes in AM. Furthermore, archival analysis was carried out to identify the current organisational strategy of Company X for transitioning into BIM-based workflows. A number of project documents including the discovery workshops approach, strategic implementation guide and white papers were also reviewed. Figure 2 below shows the methodological process of the study.

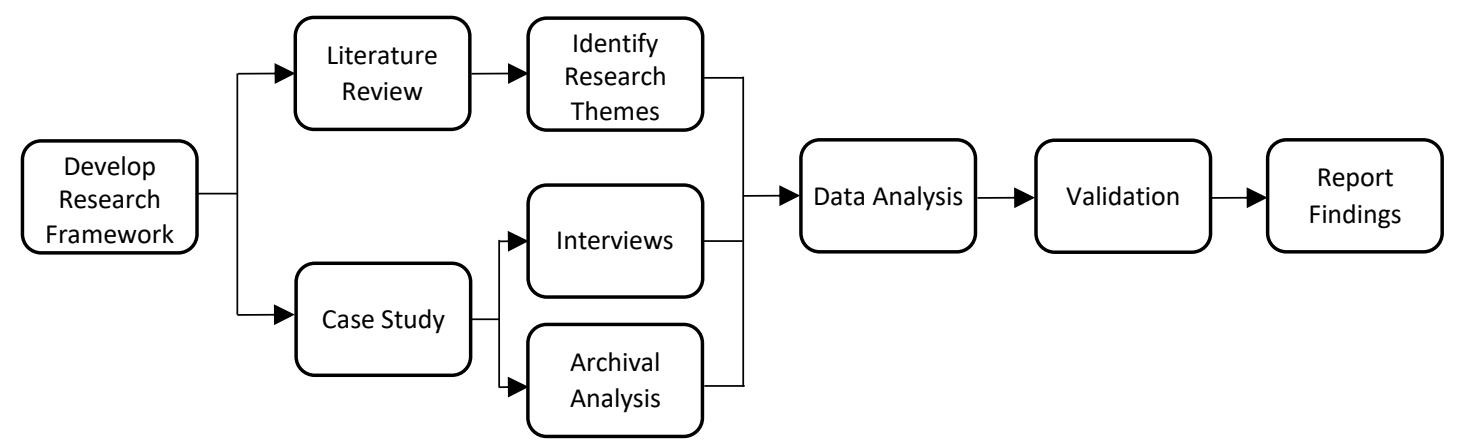

Figure 2: Research Methodology

\subsection{DATA COLLECTION}

The study adopts a qualitative approach towards data collection and analysis. Qualitative research methods elicit information on people's experience, feelings and priorities (Saunders et al., 2012). Furthermore, the qualitative approach allows researchers to probe the research questions with the aim of gathering information (Fellows and Liu, 2015). The study utilised qualitative methods in order to document the strategic approach for the standardisation of the asset register, asset hierarchy, asset information model and business processes for streamlining AM systems towards BIM-based integration. Primary data was collected through interviews and archival analysis. Interview data was transcribed and analysed using the Nvivo software. The study utilised thematic analysis in data analysis. Thematic analysis is a technique used in qualitative research for identifying, analysing and reporting themes or patterns within data (Boyatzis, 1998). Initial themes were established from literature, which guided the various aspects of data analysis for this study. That is, governance, change management, stakeholder management and performance management. The study utilised the technique of coding for easy analysis of data and linkage to the study themes. Coding in qualitative analysis is a method of indexing data to facilitate easy retrieval and analysis (Boyatzis, 1998). The study focuses on Company X's organisational BIM-AM strategy, 
BIM-AM implementation strategies, BIM-AM implementation process, BIM-AM business value and BIM-AM barriers. These notations were developed as part of the thematic analysis done in the literature review phase. The themes informed the development of interview questions for data collection. The interview questions guided coding and categorisation of data during analysis. The study characterised the themes into codes, categories and notations as shown in Table 1 below:

\begin{tabular}{|c|c|c|c|c|}
\hline $\mathbf{S} / \mathbf{N}$ & THEME & CODE & CATEGORY & NOTATION \\
\hline \multirow[t]{3}{*}{1} & \multirow[t]{3}{*}{ Governance } & $\begin{array}{l}\text { Data Governance } \\
\text { Strategic Governance }\end{array}$ & Section 4.5 & Governance \\
\hline & & $\begin{array}{l}\text { Data Governance } \\
\text { Strategic Governance }\end{array}$ & Section 4.6 & Asset Register \\
\hline & & Strategic Governance & Section 4.7 & Asset Hierarchy \\
\hline \multirow[t]{3}{*}{2} & \multirow{3}{*}{$\begin{array}{l}\text { Change } \\
\text { Management }\end{array}$} & Organisational Strategy & Section 4.2 & BIM-AM Strategy \\
\hline & & Operational Strategy & Section 4.3 & $\begin{array}{l}\text { BIM-AM } \\
\text { Implementation }\end{array}$ \\
\hline & & Systems Strategy & Section 4.4 & $\begin{array}{l}\text { BIM-AM Systems } \\
\text { Architecture }\end{array}$ \\
\hline 3 & $\begin{array}{l}\text { Stakeholder } \\
\text { Management }\end{array}$ & Stakeholder Engagement & Section 4.3 & $\begin{array}{l}\text { BIM-AM } \\
\text { Implementation }\end{array}$ \\
\hline \multirow[t]{3}{*}{4} & \multirow[t]{3}{*}{$\begin{array}{l}\text { Performance } \\
\text { Management }\end{array}$} & $\begin{array}{l}\text { BIM Value Management } \\
\text { Process }\end{array}$ & Section 4.3 .3 & $\begin{array}{l}\text { BIM-AM Benefits } \\
\text { Realisation }\end{array}$ \\
\hline & & $\begin{array}{l}\text { BIM Business Value/ } \\
\text { Operational Merits }\end{array}$ & Section 4.7 & BIM-AM Benefits \\
\hline & & Operational Demerits & Section 4.8 & BIM-AM Challenges \\
\hline
\end{tabular}

Table 1: Shows data themes, codes, categories and notations 


\subsection{CASE STUDY: BIM-AM SYSTEMS INTEGRATION AT COMPANY X}

\subsection{INTRODUCTION}

Company $\mathrm{X}$ as an asset owner and owner-operator has set out on an initiative to implement BIM-based processes in their organisation. This action aims to position Company $\mathrm{X}$ as of the leading owner-operators to utilise BIM in AM processes in the UK. The utilisation of BIM in their AM processes will improve efficiency and give the organisation an advantage over their competitors. This transformation enabled documents and data to be accessed from any facility through a data infrastructure that is supported from multiple locations. These processes are expected to improve information delivery within Company $\mathrm{X}$ for critical decision making and better management of its built assets.

Company X had engaged consultants, contractors and their supply chain who have the ability to work with industry collaborative standards and intelligent object modelling. Also, they engaged consultants to advice on best practices and standards that will contribute to success in implementation. Thus, appraising asset data architecture for integrating new systems and planning change management activities in line with the organisational goal of attaining BIM Level 3.

Company X started the journey by modelling its assets and reaching BIM Level 2. They further set the target of reaching BIM Level 3 on its project level documentation and AM processes. That is, to fully integrate its data and systems architecture for optimal information management and data delivery. Currently, Company X have modelled about 100 of their new assets and are still implementing 3D-BIM for new developments and major upgrades. However, there has been a shift in strategy and Company $\mathrm{X}$ is now focused on realigning AM processes to BIM-based processes using 2D object-based CAD. This strategy was adopted predominantly for existing buildings because of the high cost of digitising the built assets in 3D compared to the achieved benefits.

Initially, Company X viewed BIM from the perspective of 3D Modelling of their assets but the organisation's focus is gradually shifting to building information management. That 
is, looking at how information from their assets can be sourced, managed, stored and retrieved efficiently for AM tasks through BIM-based processes.

\subsection{BIM-AM STRATEGY}

In formulating an implementation strategy, Company X developed a three-part rationale for their BIM-AM based integration. The plan of action set out to establish a system that could find asset information within the organisation and across different systems quickly, accurately and completely through better management and continued development of information and systems. Company X were able to develop maturity levels for their data and systems development, but ultimately, the vision is to have single source data for their entire AM processes. Company $\mathrm{X}$ is currently working with all departments to remove data duplications across their systems and ensure that every asset has the same unique ID across the database. As the maturity grows, it is expected that for all their assets, the central AM system would generate the asset name and numbers from a single source.

The BIM-AM strategy for Company X consists of four components, which are: the right systems; defined data; culture and training; and the right process. The selection of the right systems for AM tasks is vital to the entire process. These systems will provide a platform for data exchange within the organisation. Defined data refers to the processes involved for the organisation to identify and understand the data requirements for each department and user in the whole organisation. Culture and training involves the continuous capacity development of the users and the change management strategies utilised for effective implementation. The right processes in this context represent the entire business processes re-engineering activities for optimum efficiency. In general, it is expected that this strategy will provide Company $\mathrm{X}$ a mechanism that will deliver three things for the organisation - a filing cabinet, a database and a process manager ( 3). That is, a data structure to organise their data, a secure and efficient system architecture to store it, and a structured workflow to guide its internal processes. 


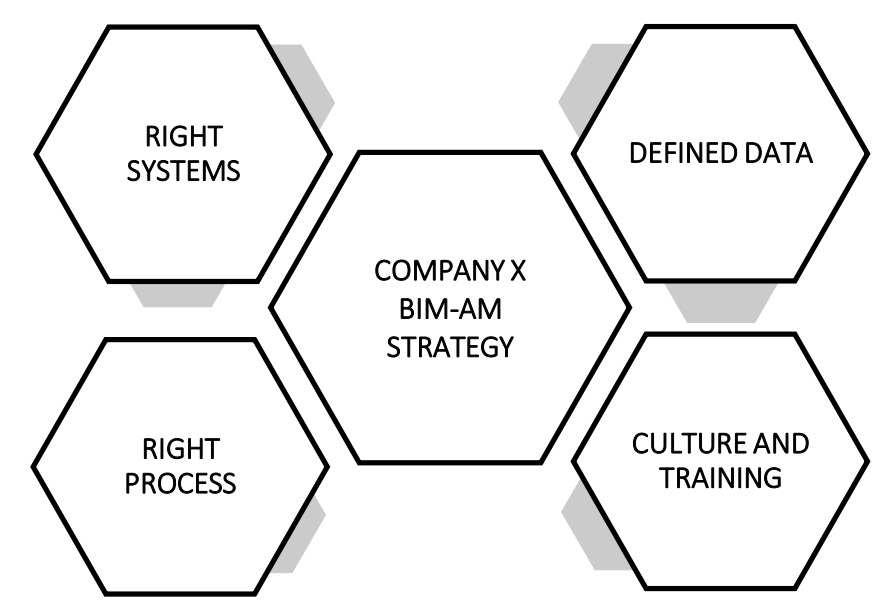

Figure 3: Company X BIM-AM strategy

\subsection{IMPLEMENTATION OF BIM-BASED PROCESSES IN AM}

The implementation process follows the strategy formulation, and one of the first activities that Company X conducted was to develop a set of Plain Language Questions (PLQs) to help drive their BIM-AM strategy. These PLQs ask the very basic queries like: 'Have we got any...?'; 'How many...?'; 'Where are ...?'; 'When is the...?'; 'Is there...?'; 'Which...?'; 'Who is...?'; within the organisation. Furthermore, Company X developed cartoon characters that asked the same PLQs for their workshops to aid understanding. These characters depicted the typical working environment in Company $\mathrm{X}$, interacting and conversing in plain language asking and answering basic queries.

The next step Company X took was to develop organisational standards for data exchange and information delivery. Company $\mathrm{X}$ developed their organisational standards based on BS:1192 (BSI, 2016), PAS:1192-2 (BSI, 2013), PAS:1192-3 (BSI, 2014a), BS:1192-4 (BSI, 2014b), and PAS:1192-5 (BSI, 2015). These standards govern data use at Company X.

The implementation process involved three phases; BIM-AM strategic stage, BIM-AM implementation stages and BIM-AM benefits realisation stage. As an implementation activity, the discovery exercise is a stand-out activity where the organisation investigates and understands the daily tasks of its employees and various departments together with 
their objectives and needs. This is a key process as it helps define all subsequent activities involved in the implementation process.

\subsubsection{BIM-AM STRATEGIC STAGE}

The strategic phase involves four essential processes, which are; discovery phase, outline business case, proof of concept and full business case (Figure 4).

\section{BIM-AM STRATEGIC STAGE}

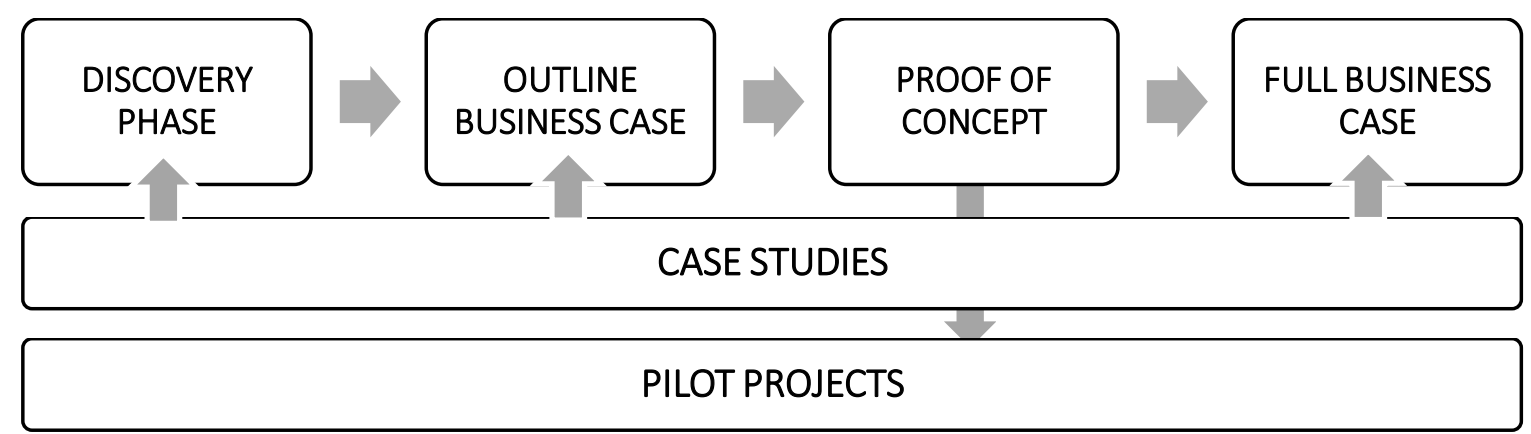

Figure 4: The processes involved in the strategic stage

The implementation process and strategic stage starts-off with the discovery exercise. The discovery methodology adopted by Company $\mathrm{X}$ essentially involves talking to users and asking the right questions about their data requirements for daily tasks. This process aims to identify information needs of users and eliminate duplication of tasks within the organisation. The process enables the team to investigate deeply as to what data source provides information for which task, the different types processes involved in executing a particular task, and the outcomes that are intended. This is usually a one-off process for every AM-personnel and a repetitive process for every department. Thus, while conducting the discovery across departments, any incongruity observed by the team is turned into case study. The full list is filtered and a shortlist is drawn for pilot projects.

The discovery exercise involves five key components, which are; desktop discovery, stakeholder engagement, stakeholder discovery, stakeholder workshops, and technical and data source discovery (Figure 5). Desktop discovery involves the review of any publicly available documents and information. Organograms, strategic documents and 
business processes are studied by the implementation team. This activity is carried out to fully understand the nature of the organisation. Stakeholder engagement concerns the process of liaising with other departmental teams at Company $\mathrm{X}$, in order to communicate on the overall vision for the information management processes, the associated impacts to business processes, and the proposed data governance structures. This activity also focuses on the needs and targeted benefits for the BIM-based processes. Stakeholder discovery involves the capturing of high level systems and process information in Company X. This process will identify key personnel that are responsible for the capture, production, utilisation and transmission of information within the organisation for the effective management of assets. Managers will be called in to participate in identifying and classifying key activities and subsequent recommendations for potential improvements. Stakeholder workshops are conducted to identify users' perceptions of the current information flow processes which will guide the roadmap. Work shadowing is also conducted at this stage. This main purpose of this activity is for external observers to identify any obstacles and work-around that are not easily identifiable during the interview workshops. Technical and data source discovery involves the development of high level 'as-is' process maps of the current systems used by departments across Company X. These documents will be developed into process maps to fully understand the system intricacies and data flow between users, systems and departments. All the five components of the discovery exercise mentioned above probe four main elements organisation-wide. These are the data sources, processes, systems and users' requirements.

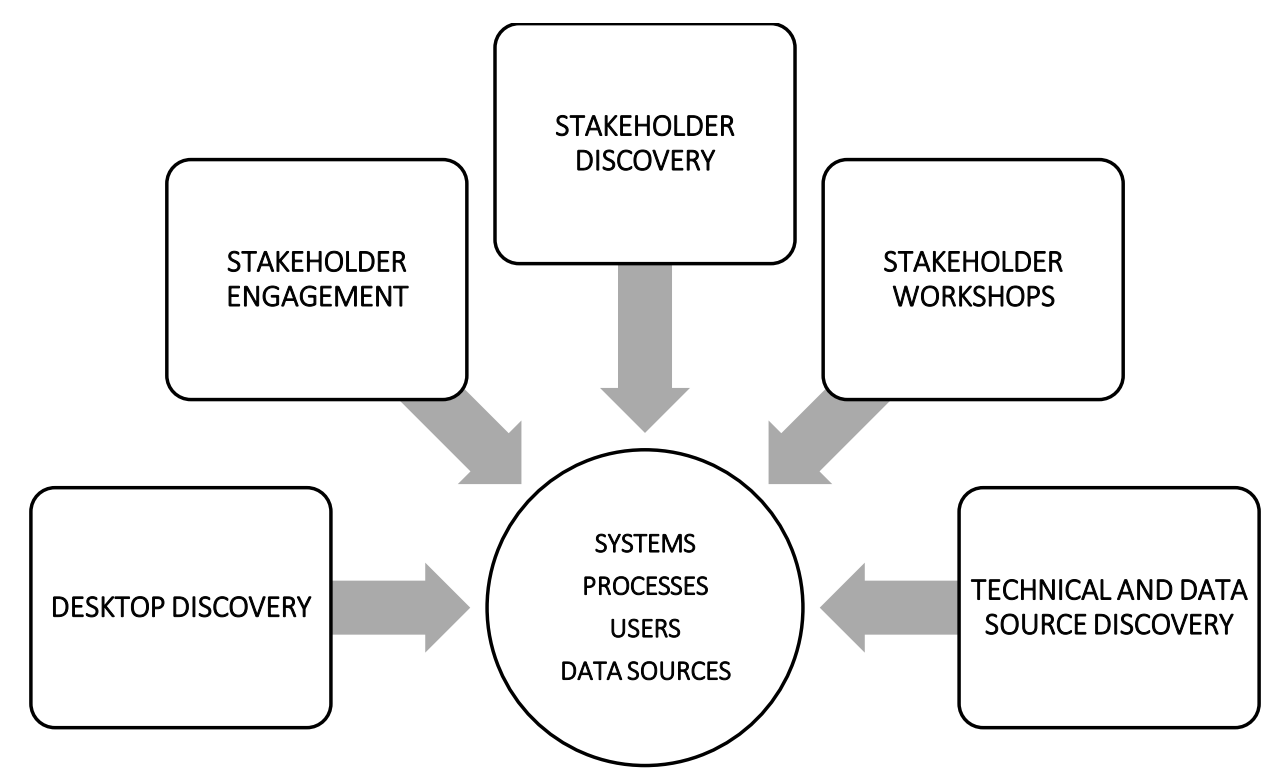

Figure 5: The key components of the discovery exercise 
The next phase after discovery is the development of the outline business case. The organisation prepares the business case on the basis of its expected commercial benefits of adopting BIM-based processes. At this level, the organisation can appraise preliminary results of the benefits realisation activity conducted on the identified case studies and pilot projects from the discovery exercise. These benefits will then be fed back into the outline business case.

Following the outline business case is the proof of concept. In this context, the tasks carried out involve the testing of BIM in AM processes with the aim of establishing viability, isolating technical issues and identifying indicators that may guide implementation. The information derived from the discovery exercise will help shape the proof of concept that will drive the experimentation in pilot projects.

The full business case involves the preparation, acknowledgement and approval of the developed business case. This is based on the discovery exercise, case studies and pilot projects. The business case is finalised by the implementation team and signed-off by the board.

\subsubsection{BIM-AM IMPLEMENTATION STAGE}

Many asset owners that aim to implement BIM often start from this stage and totally ignore the strategic phase. Usually, they make the BIM investment and then work out how the existing systems will be configured. Company X had developed and implemented a strategic phase that will enable the organisation to prepare properly for the implementation stage. At this stage, the lessons learnt from the pilot projects help guide implementation. The implementation stage consists of three main processes; detailed design phase, mobilisation phase and transition phase (Figure 6).

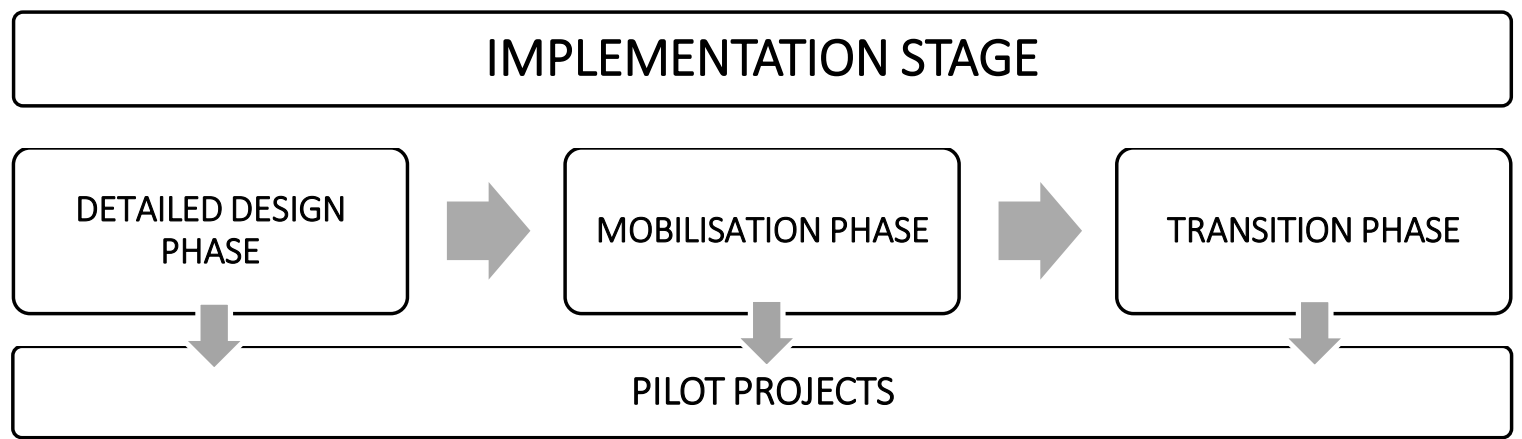

Figure 6: The processes involved in the implementation stage 
The detailed design phase involves the creation of artefacts, development of documents and configuration of systems for the BIM process as identified in the strategic stage by the implementation team. The requirements from the discovery exercise are transformed to an organisational system design document, which appropriately describes the design of the proposed BIM-AM system. It describes details of the systems, the interaction between each component, the interfaces, and its functions. Furthermore, the hardware and network approaches for the solutions from the business case are developed. As the pilot projects carry on, Company X increasingly learned from them and they fed back to the process during the development of detailed designs for the remaining case studies.

The mobilisation phase includes the processes of putting in place technical and organisational mechanisms for the deployment of BIM-AM systems at Company X. Here, Company X gradually introduced the new BIM-AM systems within the organisation to be able to control the level of disruptions to activities. From, this point the organisation starts switching to the new systems for AM tasks.

At the transition phase, Company X moved from the old systems to the new systems. Here, Company X observes the effect of the changes to the system and how users adapt to the new processes.

\subsubsection{BIM-AM BENEFITS REALISATION STAGE}

The main purpose of this phase is to realise value. An organisation should not develop a business case and be unable to measure it. This stage is important to the entire implementation process because this is where the organisation establishes project success. The benefits realisation phase involves two basic processes; result appraisal and benefits realisation (Figure 7).

\section{BENEFITS REALISATION STAGE}

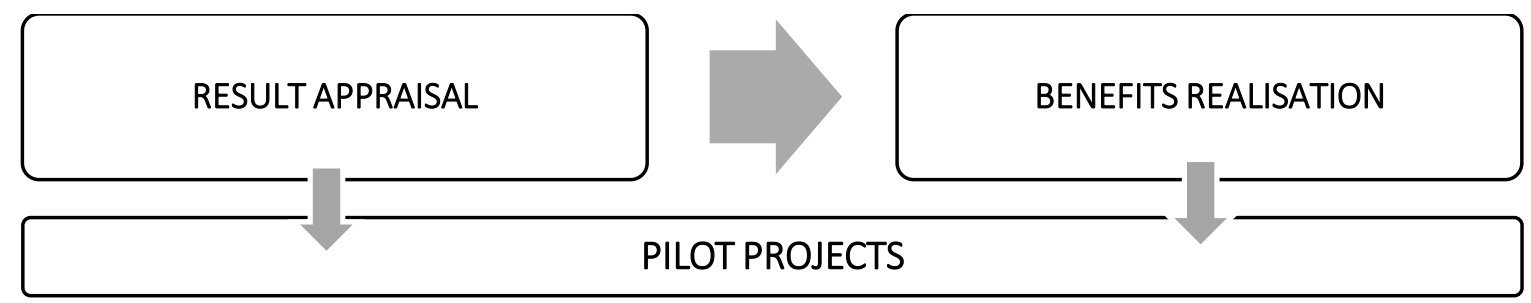

Figure 7: The processes involved in the benefits realisation stage 
Firstly, Company X had to go back to the users to observe and ask how different the processes were in order to identify the desired and undesired effects. The outcomes of change brought about by BIM had to be carefully studied. These are done in the form of questionnaire results to track the reduction in mistakes and referring back to the case studies that were developed during the discovery exercise. The outcomes are compared to the case studies for result evaluation.

Finally, the benefits are realised through established Key Performance Indicators (KPI). Another process used by Company $\mathrm{X}$, which is the deployment of questionnaires to appraise users' perception before and after implementation. The outcomes are compared for any positive or negative variance. The entire results established at this stage are properly documented. Benefits realisation encompasses the acknowledgement of the value derived from the BIM-based processes.

\subsection{BIM-AM SYSTEMS ARCHITECTURE}

The systems architecture in Company $\mathrm{X}$ is unique with a variety of platforms for executing the multifarious business processes as shown in Figure 8 below. For AM processes, Company $\mathrm{X}$ utilises an interface for case and task management with automatic routing and escalating important events or tasks 'System A'. This is the main repository that generates and holds the master asset ID, from which the other systems obtain information. Also, Company X uses a property, asset, facility and financial management platform in the operation of its asset portfolios 'System B'. This is used to monitor assets, manage compliance with regulations, streamlining invoicing, and managing asset interventions related to rates, rents and leases. Furthermore, Company X deploys a facilities management system 'System C' for the day-to-day operation of their assets. This system holds most of the information in the asset register. Also, Company $\mathrm{X}$ utilises an information management and project extranet system 'System D'. This provides a platform for securely storing and managing the project asset data. Finally, 'System E' which is essentially the use of spreadsheets, to manage data from external sources for the purpose of centralisation.

For BIM-based processes, Company X utilises a 3D modelling software 'System F'. Also, Company X uses a computer aided design software 'System $G$ ' for the development of 2D 
object-based CAD in their BIM processes. 'System G' is operated with a plug-in 'System H', which is essentially a placement tool and ensures standardisation of asset floor plans. 'System I' is the CDE. Also, Company X utilises a construction collaboration platform called 'System J'. This is a web-based platform that helps bring BIM-based data into one platform. This enables Company X to plan, implement and deliver fully integrated BIMbased build programmes.

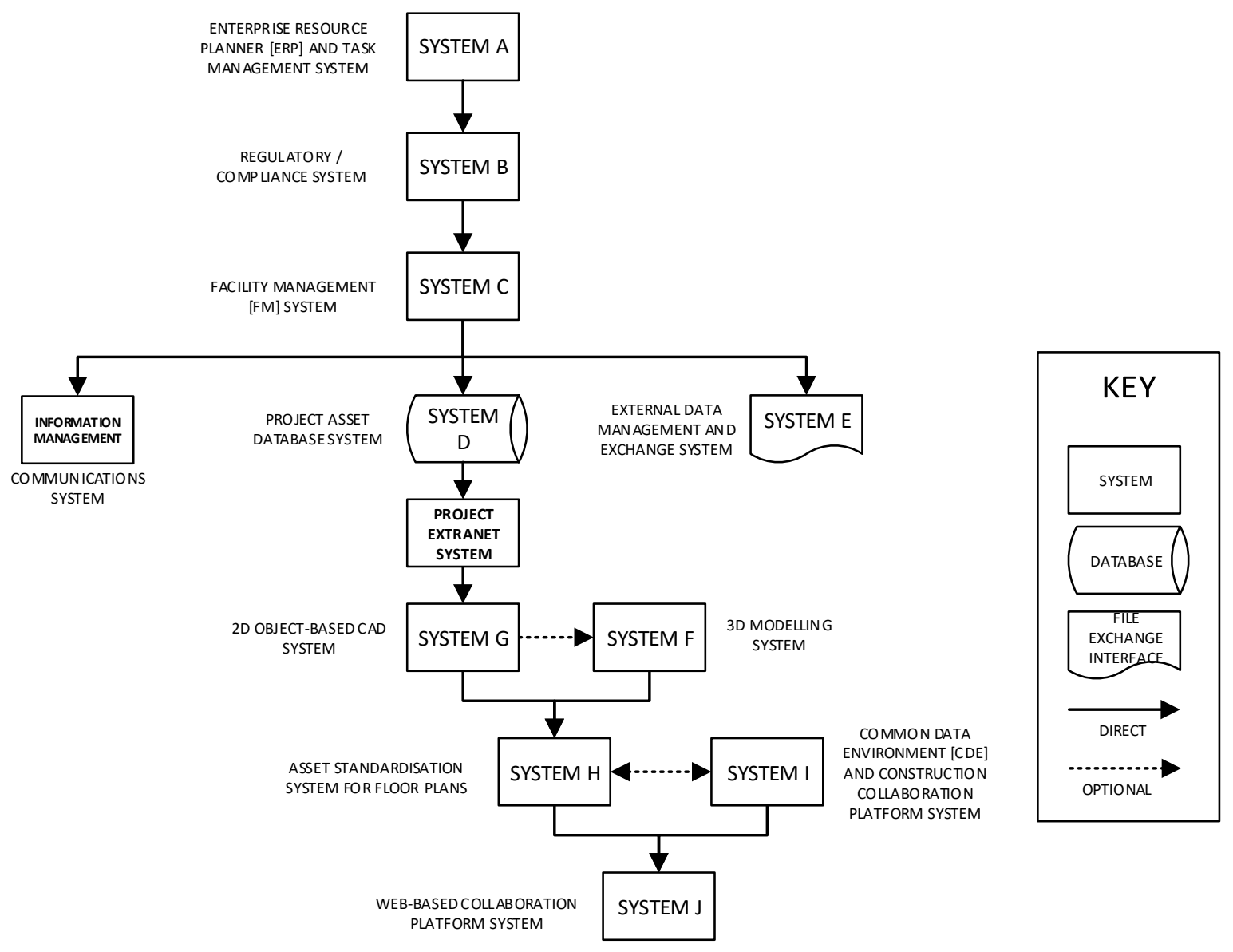

Figure 8: Systems architecture at Company X

\subsection{GOVERNANCE}

The philosophy of data governance in Company $\mathrm{X}$ is ensuring that users are engaged continuously throughout the change process of adopting new information management processes including process modifications relating to how information is created, stored and shared. The standards mentioned in 4.3 above were used to develop the new approach of information management, document control, data governance and data auditing in Company X. 
Information transaction standards were developed to guide the new information architecture for BIM-based AM processes. These standards have defined data structures, picklists, find fields and classifications to ensure that an asset is captured and referenced consistently in all systems across Company X. Assets are labelled in a systematic reference to eliminate ambiguity organisation-wide. One of the activities performed was to establish a format for the allocation of a unique identifier for every asset across Company X. Also, a file naming code sheet was introduced for asset naming and floor numbering systems during project execution and ensuring that the supply chain reference the same asset space using the unique ID allocated by Company X. This document helped in standardisation, version control and to ensure every user calls an 'apple' an 'apple' right through the entire process.

Another key aspect to data governance is the centralisation of data, which Company X associates with data completeness. Company $\mathrm{X}$ realised that centralisation of data is important and quality decisions on assets can only be made if the data available is complete. Company $\mathrm{X}$ found out that individual data managed offline by users may be of benefit to their colleagues for executing other tasks within the organisation. They are now making efforts to bring together all data in one centralised location.

Furthermore, another aspect related to governance is the technical aspect of systems development synchronisation. This activity was done through the utilisation of Application Programming Interfaces (API) to make AM processes more efficient in Company X. These sets of definitions, tools and protocols help connect the various databases to enable reporting across them. This involves defining processes and making sure that other remote systems gets updated with the right information, at the right time, with no duplications.

Lastly, another aspect related to governance is the organisational structure. There is a department responsible for regulating the data standards and practices for data governance at Company $\mathrm{X}$. It is a department under the property division. This department also liaises with the FM team within the organisation for the effective management of assets within the organisation. 


\subsection{ASSET REGISTER}

Company $\mathrm{X}$ has a standard process for developing the asset register which is essentially a property list. The register has many classifications based on ownership, asset type, location and asset function. Company $\mathrm{X}$ has a standard process at key milestones of every project to the point of handover. The processes start with the client brief to the point of obtaining planning permissions. It is at this point that the organisation decides on whether the asset will be built. In general, the entire processes adopted by Company X are in line with the RIBA plan of work. All asset information collected right through the asset development process is stored in 'System A'. This system is a central repository that holds everything and manages the process of asset development up to the point where it is assigned a unique ID to an asset.

Essentially, when the asset obtains a planning permission, it gets a unique ID. This ID will be based on the asset classification in the asset register. This classification generates many other individual data for different departments in the organisation which is stored in 'System C'. The various departments then continue their activities to develop the asset register further. For example, the FM team will populate the built asset register with other physical assets such as furniture, equipment, fittings and fixtures. The process is usually done with stickers and a handheld scanner to take inventory for the asset register.

In the asset register, most assets have an asset location ID, which is the classification of a group of assets at a particular site. This unique ID reflects the hierarchy classification of the asset, which is usually based on its function, type and location. Furthermore, other details are recorded in the register, which include functional areas of the asset such as sales floor, concessions, warehouse holding and customer facilities areas. This activity is done so that the business is able to track the unusable areas in all of its assets from the asset register.

Another system, 'System B' holds the data for the non-trading assets in the organisation. 'System A' is linked to 'System B' which is an outsourced property management solution that is managed by a third party. This system looks after rates, rents and everything else that is non-trading. 'System C' also holds the register for the remaining physical assets. These three systems together give the full asset register for the organisation (Figure 9). 


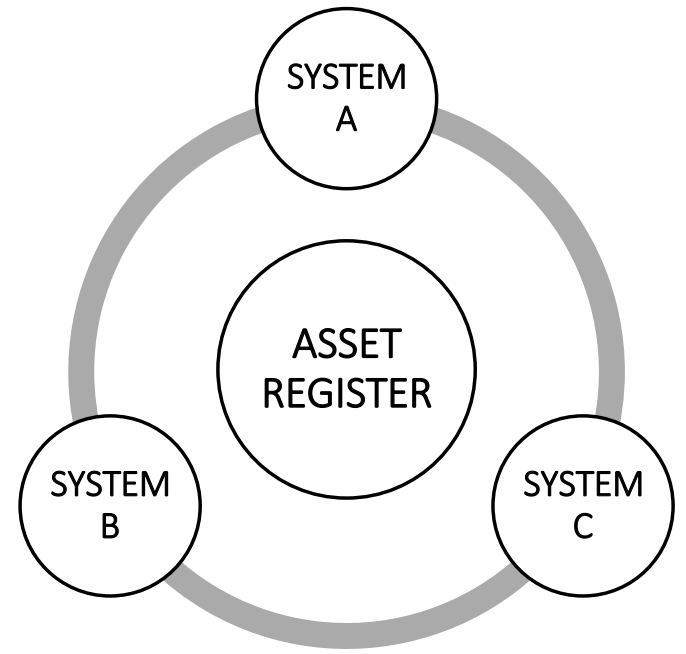

Figure 9: Systems that make up the asset register at Company X

\subsection{ASSET HIERARCHY}

Company X has developed an asset hierarchy that goes from the general to the specific. The major classifications are; business zones, business region, location, assets, levels, rooms and spaces, and components (Figure 10). The asset is given a particular reference for any of the classifications it represents. The business zone is the defined operational zones within the organisation. The business region is a collection of sites within a business zone. The location is the particular site where the asset is situated. The asset represents the type of building and the function that it performs. Levels within the asset hierarchy show the number of levels contained in an asset. This deals with occupancy and space management. Rooms and spaces represent the individual spaces within an asset. This concerns the types of areas within the asset such as customer facilities or trading floor. Components are the physical assets that have been installed within a particular space. The component type and other parameters are recorded at this level. The hierarchy is defined for every asset and corresponding areas and components within them.

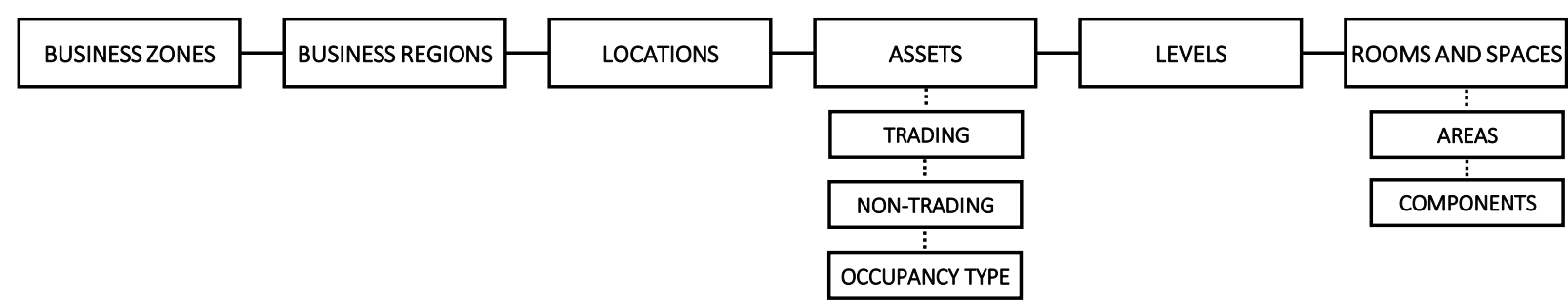

Figure 10: Asset hierarchy structure of Company X 


\subsection{BENEFITS OF BIM-AM SYSTEMS INTEGRATION}

Company $\mathrm{X}$ has been able to derive a number of benefits from the BIM process. The use of 3D-BIM offered visualisation, communication and coordination as benefits for the construction processes. Also, another benefit derived was that products can be linked to sales performance and costs items can be viewed visually in 3D.

For the AM processes, BIM has enabled Company $\mathrm{X}$ to effectively manage its asset information through better information management, document control, governance, and auditing. Another benefit derived is the standardisation of processes organisationwide. The utilisation of 'System J' provided a platform for Company X to manage the specifications, standardisation of components and room datasheets.

\subsection{CHALLENGES OF BIM-AM SYSTEMS INTEGRATION}

From the interviews conducted, the following themes were identified as barriers to the implementation of BIM in AM; they are; complexity and cost associated with BIM, irrelevance of 3D spatial data in AM processes, ownership structure of assets, managing the asset handover process and managing change within the organisation.

\subsubsection{COMPLEXITY AND COST ASSOCIATED WITH BIM}

The respondents highlighted the high cost of updating and maintaining the 3D-Models as a barrier to implementation. It was highlighted that the organisation would have to be sure of the savings to be achieved before they would embark on digitising the existing asbuilt assets to 3D models. Furthermore, the interviewees indicated that at the moment they have not found any evidence of real value for converting existing documentation to 3D. Also, the respondents mentioned that maintaining updated as-built 3D models would require a lot of resources and that it would be a huge investment to do the conversions.

The respondents further stated that their initial 3D-BIM approach did not work for them as the consultants paid a lot of attention to the design and construction models. Essentially, the models contained too much information that they did not need for the purpose of AM tasks. The respondents concluded that currently there is organisation preference of 2D object-based CAD for AM processes. The availability of 3D-goemetric 
data was an additional benefit to the organisation, but considering the cost, 2D objectbased CAD provided better resource efficiency and cost effectiveness.

To support these findings, McArthur (2015) highlights the difficulty associated with digitising as-built assets and the high level of effort required to create new or modify existing BIM models for built assets.

\subsubsection{IRRELEVANCE OF 3D-GEOMETRIC DATA IN AM PROCESSES}

One of the key findings of this study is the assertion by respondents that 3D-Geometric data may not be very relevant for AM tasks. The respondents stated that during the process of strategy development, Company X realised that 3D-models were of no value to their operations. Furthermore, the respondents highlighted that what the users needed was asset information on floor plan layouts which could be delivered with 2D-objectbased CAD. Hence, the switch in focus by the organisation from 3D-BIM to an information management perspective, which focused more on 2D object-based modelling. Company $\mathrm{X}$ derived benefits from the use of BIM such as retrieving asset data and product sales visually, but the respondents highlighted that regardless of BIM, they had the capability to evaluate their products' performance data using existing systems, and all BIM contributed was accessing the information visually.

Generally, the requirements for AM tasks are not properly understood at the front end of the project delivery process. As a result, data handed over from the design and construction phases must be filtered down so that asset managers get the data they actually need for their daily work.

In line with these findings, Mayo and Issa (2014) suggest the requirement of nongeometric information for business processes in the operations and use phase.

\subsubsection{OWNERSHIP STRUCTURE OF ASSETS}

Another barrier is the ownership structure of assets. Every owner-operator has its unique organisational structure including its business structure, which determines how assets are owned, operated and maintained. The interviews revealed that Company X did not own some of their assets, as a number were leased or concessioned. This reason alone 
makes it difficult to implement BIM for those assets as they would not be operating them. Without total ownership of the assets, it may not be of much value for Company $\mathrm{X}$ to invest in BIM in those circumstances.

\subsubsection{MANAGING THE ASSET HANDOVER PROCESS}

An important factor that affects the implementation of BIM in AM is the manner in which the handover process is managed. The respondents asserted that updating the asdesigned models to include every as-built information required a lot of resources. They also highlighted that the organisation had not put in place any mechanism to validate the 3D models at hand over stage.

In line with these findings, Jupp (2013) suggest a lack of a holistic approach to BIM and lifecycle management which include a lack of procedures for verifying the as-built datasets as a barrier for the operations and use phase. Furthermore, Jupp (2013) highlights the challenges for utilising BIM models at handover as a result of incomplete models, lack of verification of models, lack of capture of requirements, knowledge and expertise for the operations and use phase. Also, McArthur (2015) highlights the challenges of handling uncertainty where building documentation is incomplete at handover.

\subsubsection{MANAGING CHANGE WITHIN THE ORGANISATION}

Change management within the organisation is a significant factor towards BIM implementation in AM. The respondents revealed that the organisation experiences some challenges in the consistent management of the roll-out process and adoption organisation-wide. Furthermore, the respondents mentioned that the culture of adoption and change perception of users is an aspect that the organisation is focusing on in order to develop solutions. The interviewees pointed out that some of the solutions were realised from the stakeholder workshops and shadowing activity conducted during the discovery exercise. They further highlighted that since designers at the front end do not understand the nature of activities the asset managers do on a daily basis; the discovery and subsequent recommendations focus on current work issues and proffer more simplified processes that will make AM tasks more efficient. 
Consistent with these findings, Jupp and Awad (2017) identify challenges of transitioning from traditional to BIM-based processes as a barrier to implementation. These management shortcomings were also identified by Jupp (2013) stating that the integration and management of processes, technologies and protocols across building lifecycle phases continues to present major challenges to its implementation. 


\subsection{CONCLUSION}

This study explored the strategic and operational techniques of implementing and streamlining BIM with AM systems, and how it can be achieved by an asset owner through a research case study. It describes in detail organisational strategies of BIM-based integration in AM including the strategic approach and implementation plans. Specifically, the study identifies the 'discovery phase' as a key process in identifying what information should be captured from physical assets towards BIM-based integration. The study also highlights the business value of BIM-based integration in AM including the benefits management realisation process. The findings of this study will help guide asset owners seeking to implement BIM in AM and realise business value from BIM-AM systems integration.

Furthermore, the study clearly confirms that one of the reasons of the slow adoption of BIM in AM is the industry perception of BIM, which is seen as a 3D-model, not as an information model or information management tool. Also, the paper clears the misconception of having all asset data in one model or system as a prerequisite of implementing BIM. The information can be in several systems provided that they are all linked.

Company $\mathrm{X}$ has been successful in implementing BIM and integrating it with their AM systems. For an organisation to be successful in streamlining asset data with BIM systems, the following key issues have to be considered: the development for a clear strategy prior to adoption; connecting the strategy to the business goals; and conducting the discovery exercise to identify organisational information needs. Generally, to overcome BIM adoption barriers in AM, asset owners need to understand their organisational information needs for AM tasks. They also need to understand the type of organisational change required in adopting BIM-based business processes. Owneroperators that have been able to streamline their AM and BIM systems did so through discovery of data needs, development of in-house standards, and continuous improvement of management practices that govern such systems. This also included management activities such as standardising assets within the register with unique identifiers, understanding the business processes and the information needs of users, 
business process re-engineering, and recommending the right systems to support the digital workplace. 


\section{REFERENCES}

Ashworth, S., Tucker, M. and Druhmann, C. (2016) 'The Role of FM in Preparing a BIM Strategy and Employer's Information Requirements (EIR) to Align with Client Asset Management Strategy', In: Proceedings of CIB World Building Congress, Vol. 5.

Boyatzis, R.E. (1998) Transforming qualitative information: Thematic analysis and code development, $1^{\text {st }}$ edition, Thousand Oaks: Sage Publications, Inc.

Brous, P., Herder, P. and Janssen, M. (2015) 'Towards modelling data infrastructures in the asset management domain', Procedia Computer Science , Vol. 61, No. 1, pp. 274280.

Brous, P., Herder, P. and Janssen, M. (2016) 'Governing asset management data infrastructures', Procedia Computer Science 9, Vol. 95, No. 1, pp. 303-310, Available: ISSN 1877-0509.

BSI (2008) 'Asset Management - Part 2: Guidelines for the application of PAS 55-1 - PAS 55-2: 2008'.

BSI (2013) 'Specification for information management for the capital/delivery phase of construction projects using building information modelling - PAS 1192-2:2013', Available: ISBN: 978-0-580-82666-5.

BSI (2014a) 'Specification for information management for the operational phase of assets using building information modelling - PAS 1192-3:2014', Available: ISBN: 978-0-580-83910-8.

BSI (2014b) 'Collaborative production of information - Part 4: Fulfilling employer's information exchange requirements using COBie - Code of practice - BS 11924:2014', Available: ISBN: 978-0-580-85255-8.

BSI (2015) 'Introduction to PAS 1192-5:2015 - A specification for security-minded building information modelling, digital built environments and smart asset management - PAS 1192-5:2015', Available: ISBN: 9780580882579.

BSI (2016) 'Collaborative production of architectural, engineering and construction information - Code of practice - BS 1192:2007+A2:2016', Available: ISBN 9780580 928178.

Campos, J., Sharma, P., Gabiria, U.G., Jantunen, E. and Baglee, D. (2017) 'A big data analytical architecture for the asset management', Procedia CIRP: Circular Perspectives on Product/Service-Systems, Vol. 64, No. 1, pp. 369-374, Available: ISSN 2212-8271.

Cavka, H.B., Staub-French, S. and Poirier, E.A. (2017) 'Developing owner information requirements for BIM-enabled project delivery and asset management', Automation in Construction, Vol. 83, pp. 169-183.

Codinhoto, R. and Kiviniemi, A. (2014) 'BIM for FM: a case support for business life cycle', Yokohama, 63-74. 
DBIC (2015) The COINS Standard - Introduction, 1 Nov, [Online], Available: http://www.coinsweb.nl/COINS2.0/5 Introduction COIN \%20standard V5 Nov2 015.pdf [12 Feb 2018].

Eastman, C., Teicholz, P., Sacks, R. and Liston, K. (2011) BIM Handbook: a guide to Building Information Modeling for owners, managers, designers, engineers and contractors, $2^{\text {nd }}$ edition, Hoboken: John Wiley \& Sons, Inc.

Fellows, R. and Liu, A. (2015) Research methods for construction, $4^{\text {th }}$ edition, Somerset: John Wiley and Sons, Inc.

Guillen, A.J., Crespo, A., Gómez, J., González-Prida, V., Kobbacy, K. and S, S. (2016) 'Building information modeling as asset management tool', IFAC-PapersOnLine, Vol. 49, No. 28, pp. 191-196, Available: http://dx.doi.org/10.1016/j.ifacol.2016.11.033.

ISO 27001 (2013) 'Information technology - Security techniques - Information security management systems — Requirements — ISO 27001'.

Jupp, J. (2013) 'Incomplete BIM implementation: exploring challenges and the role of product lifecycle management functions', Nantes, France.

Jupp, J. and Awad, R. (2017) 'A change management perspective on BIM-FM implementation', 231-370.

Kotter, J. (1996) 'Leading change', Harvard School Press, Boston, MA.

Lewin, K. (1951) Field theory in social science, New York: Harper and Row.

Lin, S., Gao, J. and Koronios, A. (2008) 'A data quality framework for engineering asset management', Australian Journal of Mechanical Engineering, Vol. 5, No. 2, pp. 209219, Available: http://dx.doi.org/10.1080/14484846.2008.11464549.

Lin, S., Gao, J., Koronios, A. and Chanana, V. (2007) 'Developing a data quality framework for asset management in engineering organisations', International Journal of Information Quality, Vol. 1, No. 1, pp. 100-125.

Love, P.E.D., Matthews, J., Simpson, I., Hill, A. and Olatunji, O.A. (2014) 'A benefits realization management building information modeling framework for asset owners', Automation in Construction, Vol. 37, No. 1, pp. 1-10.

Love, P.E.D., Simpson, I., Hill, A. and Standing, C. (2013) 'From justification to evaluation: Building information modeling for asset owners', Automation in Construction Volume, Vol. 35, No. 1, pp. 208-216.

Love, P.E.D., Zhou, J., Matthews, J. and Luo, H. (2016) 'Systems information modelling: enabling digital asset management', Advances in Engineering Software, Vol. 102, pp. 155-165.

Mayo, G. and Issa, R.R.A. (2014) 'Processes and standards for BIM closeout information deliverables', Proceedings of 2014 International Conference on Computing in Civil and Building Engineering. June 23-25, 2014 , Orlando, Florida. United States, 673680. 
McArthur, J.J. (2015) 'A building information management (BIM) framework and supporting case study for existing building operations, maintenance and sustainability', 1104-1111.

Mohseni, M. (2003) 'What does asset management mean to you?', Dallas, 962-964.

Patton, M.Q. (2002) Qualitative evaluation and research methods, $3^{\text {rd }}$ edition, Thousand Oaks: Sage Publications.

Pishdad-Bozorgi, P., Gao, X., Eastman, C. and Self, A.P. (2018) 'Planning and developing facility management-enabled building information model (FM-enabled BIM)', Automation in Construction, Vol. 87, pp. 22-38.

Saunders, M., Lewis, P. and Thornhill, A. (2012) Research methods for business students, $6^{\text {th }}$ edition, Harlow: Pearsons.

Schneider, J., Gaul, A.J., Neumann, C., Hogräfer, J., Wellßow, W., Schwan, M. and Schnettler, A. (2006) 'Asset management techniques', International Journal of Electrical Power and Energy Systems, Vol. 28, No. 9, pp. 643-654.

Succar, B., Sher, W. and Williams, A. (2012) 'Measuring BIM performance: Five metrics', Architectural Engineering and Design Management, Vol. 8, No. 2, pp. 120-142.

Vanlande, R., Nicolle, C. and Cruz, C. (2008) 'IFC and Buildings Lifecycle Management', Automation in Construction, Vol. 18, No. 1, pp. 70-78, Available: https://hal.archives-ouvertes.fr/hal00639553/PDF/IFC for Buildings Lifecycle Management v17.pdf [18 Dec 2017].

Walasek, D. and Barszcz, A. (2017) 'Analysis of the adoption rate f Building Information Modeling (BIM) and its Return on Investment (ROI)', Procedia Engineering, Vol. 172, pp. 1227-1234.

Yin, R.K. (2003) Case Study Research: Design and Methods, $3^{\text {rd }}$ edition, Thousand Oaks: Sage. 